\title{
Natural variation of morphological traits in wild wheat progenitor Aegilops tauschii Coss.
}

\author{
Shigeo Takumi*1), Emi Nishioka ${ }^{1}$, Haruhiko Morihiro' ${ }^{1)}$, Taihachi Kawahara ${ }^{2)}$ and Yoshihiro Matsuoka ${ }^{3)}$ \\ 1) Graduate School of Agricultural Science, Kobe University, 1-1 Rokkodai, Nada, Kobe, Hyogo 657-8501, Japan \\ 2) Plant Germ-Plasm Institute, Graduate School of Agriculture, Kyoto University, 1 Nakajou, Muko, Kyoto 617-0001, Japan \\ 3) Department of Bioscience, Fukui Prefectural University, Eiheiji, Matsuoka, Fukui 910-1195, Japan
}

\begin{abstract}
Aegilops tauschii Coss. (syn Ae. squarrosa L.) is a wild diploid wheat species. It has a wide natural species range in central Eurasia, spreading from northern Syria and Turkey to western China. Ae. tauschii is known as the D genome progenitor of hexaploid bread wheat. The genealogical and geographical structure of variation of morphological traits was analyzed using a diverse array of 205 sample accessions that represented the entire species range. In total, 27 traits, including anther and pistil shape and internode length, were examined in this study. Large-scale natural variation was found for all examined traits. Geographically, significant longitudinal clines were detected for anther size, internode length and spike size and shape. Anthers tended to be small in accessions from the eastern region. Internodes also tended to be short, whereas spikes tended to be long in accessions from the eastern region. Spikelet density per spike tended to be high in the eastern habitats. In the process of west-to-east dispersal, Ae. tauschii underwent extensive morphological, genetic and ecological diversification that produced the variation seen among today's natural populations.
\end{abstract}

Key Words: diversification, geographical cline, morphology, variation, wild wheat.

\section{Introduction}

Aegilops tauschii Coss. (syn. Ae. squarrossa L.), a diploid self-pollinating goatgrass, is the $\mathrm{D}$ genome donor of common wheat (Kihara 1944, McFadden and Sears 1944). The genome of Ae. tauschii was brought into that of common wheat through a spontaneous species cross with tetraploid emmer wheat and subsequent amphidiploidization about 8,000 years ago (Nesbitt and Samuel 1996). Habitats of Ae. tauschii are widely distributed from north Syria and Turkey to western China in Eurasia. Ae. tauschii populations, which maintain large diversity at the nucleotide sequence level (Caldwell et al. 2004), provide a useful source for common wheat breeding. In fact, some studies have reported higher levels of genetic variability of glutenin subunits and gliadin in Ae. tauschii than in the D genome of common wheat (Gianibelli et al. 2001, 2002, Yan et al. 2003a, 2003b, Giles and Brown 2006). Ae. tauschii has been used by wheat breeders to integrate pest resistance traits into wheat cultivars (Zhu et al. 2005).

The subspecies classification of Ae. tauschii is still under discussion. Two subspecies have been described, Ae. tauschii Coss. subspecies tauschii (syn. Ae. squarrosa L. subspecies eusquarrosa Eig) and Aegilops tauschii Coss. subspecies strangulata (Eig) Tzvel (syn. Ae. squarrosa L. subspecies

Communicated by T. Terachi

Received July 21, 2009. Accepted October 30, 2009.

*Corresponding author (e-mail: takumi@kobe-u.ac.jp) strangulata Eig) based on spikelet morphology, whereas the typical forms of subspecies tauschii and strangulata are connected by a continuous range of intermediate forms (Dudnikov 1998). Some recent reports have also shown difficulty in distinguishing the two subspecies based on molecular markers and suggested a high level of gene flow between them (Dvorak et al. 1998, Dudnikov and Kawahara 2006, Saeidi et al. 2006). Two characteristics that distinguish the subspecies have been described: one is glume width to rachis segment width ratio, another is allozyme variation at the Acph1 locus (Dudnikov 2000). Our previous study showed that these subspecies form separate clusters in a graph of the first two components from principal component (PC) analysis based on nine spikelet traits and suggested that subspecies strangulata diverged from an ancestor that carried a specific chloroplast DNA type found in the western habitats. Moreover, natural variation in spikeletrelated traits showed significant longitudinal and latitudinal clines for spikelet size (Matsuoka et al. 2009). Latitudinal and longitudinal clines were also found for natural flowering time variation. The early-flowering accessions spread mainly in eastern habitats such as Afghanistan and Pakistan, implying that the early-flowering phenotype contributed to eastward dispersal and adaptation to these habitats in Ae. tauschii (Matsuoka et al. 2008).

Relatively little is known about genetic and phenotypic characteristics of the eastern and southern populations because previous studies have mainly used accessions from the Transcaucasus and northern Iran, the center of the species' 
diversity. The eastern and southern populations (i.e., those from Afghanistan, Pakistan, Central Asia and China), however, have the potential to provide materials suitable for study of the mechanisms underlying Ae. tauschii's wide ecological adaptation. Such studies may help elucidate the expanded distribution of Ae. tauschii eastward from Transcaucasus to China. In this study, we used 205 Ae. tauschii accessions covering the species range and performed a common garden experiment to analyze the natural variation of morphological traits. PC analysis of the morphological traits and phylogenetic network based on chloroplast DNA variation provided information about subspecies formation and the process of the species' westto-east dispersal. The implications of these findings for intraspecific morphological diversification are discussed.

\section{Materials and Methods}

\section{Plant materials}

In total, 205 accessions of Ae. tauschii were used in this study (Table 1). Their passport data including geographical coordinates have been provided in previous reports (Matsuoka et al. 2005, 2007, 2008). This sample set consisted of 199 accessions representing the entire natural habitat range (Fig. 1) and six accessions (AT 47, AT 55, AT 60, AT 76, AT 80, and PI 508264) representing adventive populations in the Shaanxi and Henan provinces of China. When geographical coordinates of sampling sites were not available, we estimated latitude and longitude by means of Kashmir 3D software (http://www.kashmir3d.com/) on scanned paper maps (scales $1: 4,000,000-1: 1,000,000$ ) based on locality information. For four accessions (CGN 10731, CGN 10732, CGN 17333, and CGN 10734), geographical coordinates were not estimated due to lack of locality information. In the original collection, most of the accessions were not obtained by population sampling. For each accession, we used seeds propagated from a single plant by selfing.

Chloroplast DNA haplogroups (HGs) of the 205 accessions were also previously determined based on data from biallelic single nucleotide polymorphism and minisatellite sites that were found in the flanking regions of chloroplast microsatellite loci (Matsuoka et al. 2005, 2007, 2008, 2009). Clustal W software ver. 1.8 (Thompson et al. 1994) was used for alignment of the chloroplast DNA sequences, and Network software ver. 4.112 (http://www.fluxus-engineering. $\mathrm{com} /$ ) (Bandelt et al. 1995) was used for reduced median network construction. On the basis of the network topology, four intraspecific lineages were defined as follows: the HG7 lineage (HG7 and its derivatives HG2, HG4, HG5, HG6, HG8, HG10, HG11, HG12, HG13 and HG14), the HG9 lineage (HG9 and its derivatives HG1, HG3 and HG18), the HG16 lineage (HG16 and its derivative HG15), and the HG17 lineage (HG17) (Matsuoka et al. 2008, 2009). The major HG lineage information of the 205 accessions is represented in Table 1.

\section{Analysis of morphological variation}

Seeds of the sample accessions were sown in November 2004. Plants were grown in a field of Kobe University, and the accessions were arranged in the field using a randomized design. For each accession, a single healthy plant was chosen for analysis of morphological variation. All morphological traits were measured using the three tillers of each plant that headed earliest, and the trait averages and standard deviations were calculated.

In total, 27 traits were studied, and their abbreviations are listed in Table 2. Out of them, nine traits related to the sikelet morphology, i.e. spikelet length (SpL), empty glume length (GL), empty glume width (GW), empty glume thickness (GT), empty glume height (GH), lemma length (LL), lemma width $(\mathrm{LW})$, palea length $(\mathrm{PaL})$, and palea width $(\mathrm{PaW})$, were previously studied (Matsuoka et al. 2009), and their data was used in this study. Trait measurements were done using the first, second, and third culms and spikes. The first and second florets of the central spikelet were used for estimation of the pistil and anther traits before anthesis. Awn length was measured at the three positions, the first florets of top and central spikelets (TAL and AwL, respectively) and the fourth floret of the central spikelet (AwL4). Internode length of spikelet (ILSp) was estimated as an average of internode lengths before and after the central spikelet.

The morphological trait data were statistically analyzed using JMP software ver. 5.1.2 (SAS Institute). Cluster analysis among the traits was conducted by the clustering program (http://www.tinet.org/ debb/UPGMA/) that calculated all the Pearson correlation coefficients between pairs of sets of variables, transformed these coefficients into distances and made a clustering using the unweighted pair group method with arithmetic mean (UPGMA) algorithm (GarciaVallve et al. 1999).

\section{Results}

\section{Natural variations in 27 morphological traits}

In total, 27 morphological traits were measured before anthesis. Large natural variations were found for all examined traits, especially spike-, spikelet- and floret-related traits (Table 2). In most examined traits, the maximum values were two to three times the minimum values. Two awn length traits, AwL and AwL4, exhibited large standard deviations (Table 2). Of the 27 examined traits, 19 (SL, NSp, GL, GW, GT, GH, AwL, LL, LW, AnL, AnT, PiL, PiW, PiT, PaL, PaW, MCL, MCT, and IL4) showed a significant difference in means between the subspecies (Student's $t$ test, Table 2).

Among the examined traits, the highest correlations were observed among the glume, lemma and palea traits, and the spikelet length (SpL) was closely related to the lemma and palea lengths (LL and PL) (Fig. 2A). Cluster analysis of the examined morphological traits showed strong association among the spike, spikelet and floret traits (Fig. 2B). Anther and pistil sizes were highly associated with spikelet and 
Table 1. Strain numbers and sources of the Ae. tauschii accessions used in this study

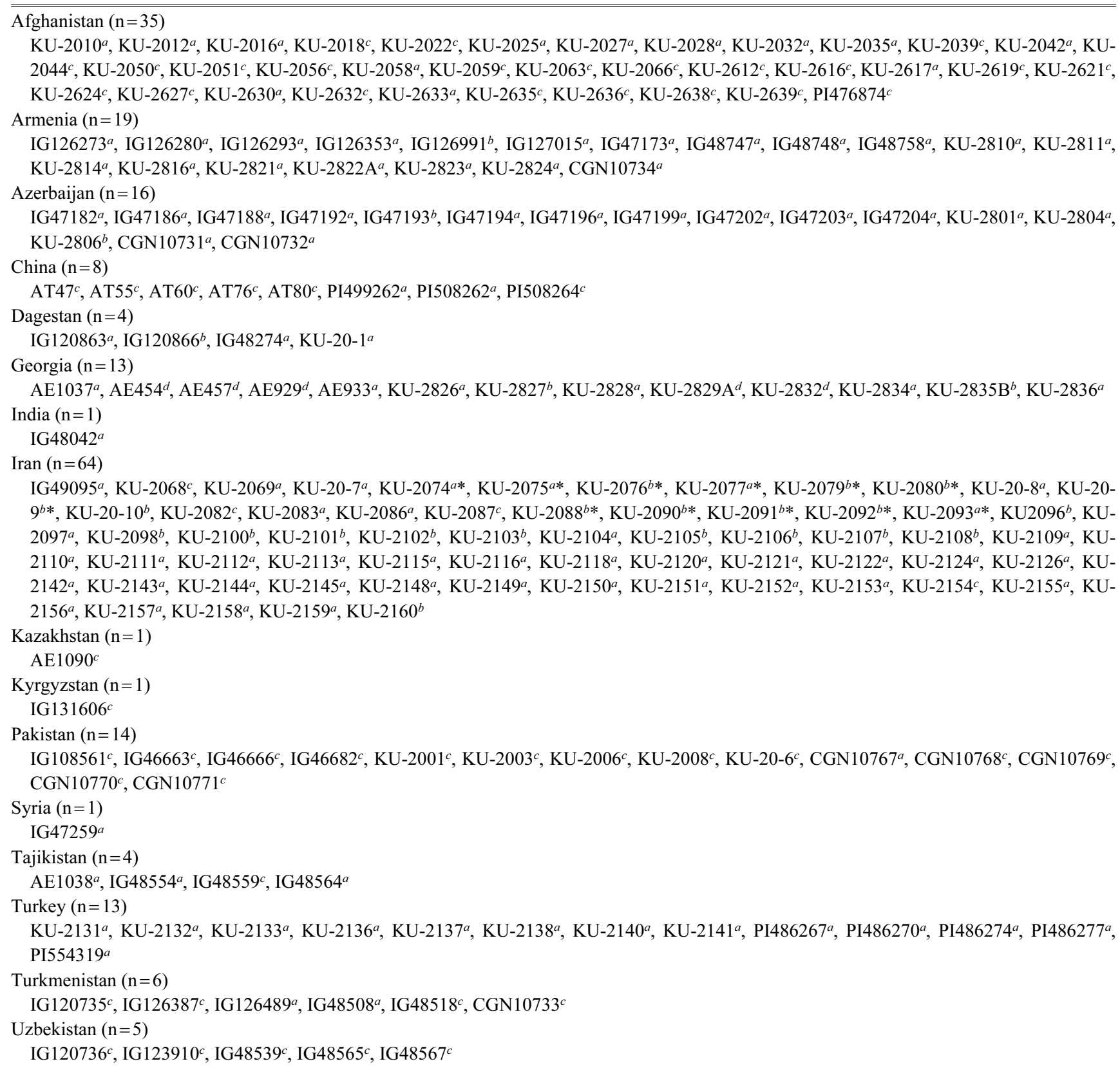

KU: Plant Germ-Plasm Institute, Faculty of Agriculture, Kyoto University, Japan. PI: National Small Grains Research Facility, USDA-ARS, USA. IG: International Center for Agricultural Research in the Dry Areas (ICARDA). CGN: Centre for Genetic Resources, The Netherlands. AE: Institut für Pflanzengenetik und Kulturpflanzenforschung (IPK). AT: Kenji Kato, Okayama University.

${ }^{a}$ HG7 lineage $(\mathrm{n}=117)$

${ }^{b}$ HG9 lineage $(\mathrm{n}=26)$

${ }^{c}$ HG16 lineage $(\mathrm{n}=57)$

${ }^{d}$ HG17 lineage $(\mathrm{n}=5)$

* ssp. strangulata $(\mathrm{n}=12)$

floret widths. Spikelet number (NSp) was grouped with spike length (SL). Culm and internode lengths were well correlated with each other, but separated from the spike, spikelet and floret traits. Awn length traits were nearly independent of other traits.

\section{PC analysis of the morphological trait variations}

To study intraspecific differentiation in Ae. tauschii, we conducted PC analysis based on the 27 morphological traits. Scatter plots with the first two PC values (PC1 and PC2) of the 205 accessions were continuous (Fig. 3A). The accumulated proportion of $\mathrm{PC} 1$ and $\mathrm{PC} 2$ in the total morphological 


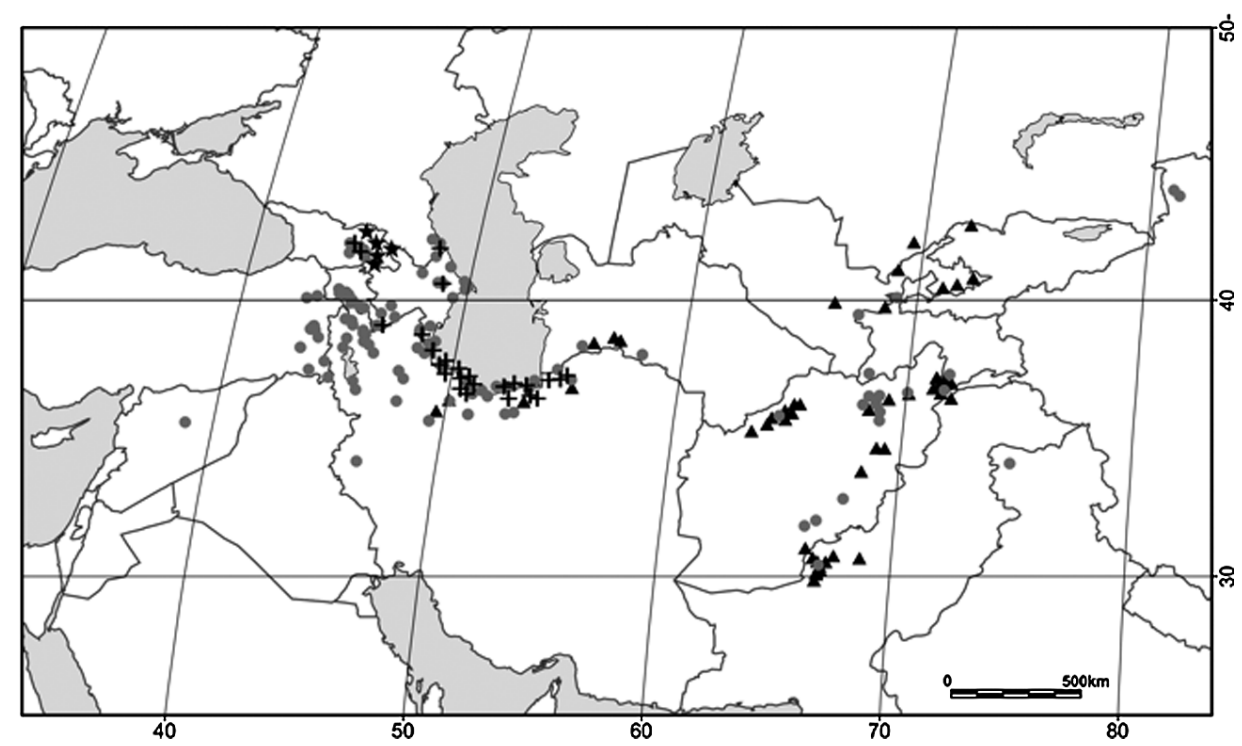

Fig. 1. Geographical distribution of Ae. tauschii accessions. Adventive populations in the Shaanxi and Henan provinces of China are not shown. Circles, crosses, triangles, and stars indicate the HG7, HG9, HG16, and HG17 lineages, respectively.

Table 2. Variation in 27 morphological traits in 203 accessions

\begin{tabular}{|c|c|c|c|c|c|c|}
\hline \multirow[b]{2}{*}{ Trait $^{a}$} & & \multicolumn{3}{|c|}{ Total accessions } & \multirow{2}{*}{$\begin{array}{c}\text { Subspecies tauschii } \\
\text { Mean } \pm \text { SD }\end{array}$} & \multirow{2}{*}{$\frac{\text { Subspecies strangulata }}{\text { Mean } \pm \text { SD }}$} \\
\hline & & $\begin{array}{c}\text { Mean } \pm \text { standard } \\
\text { deviation }(\mathrm{SD})\end{array}$ & $\begin{array}{l}\text { Maximum } \\
\text { value }\end{array}$ & $\begin{array}{l}\text { Minimum } \\
\text { value }\end{array}$ & & \\
\hline $\mathrm{SL} * * *$ & Spike length $(\mathrm{cm})$ & $11.12 \pm 1.49$ & 6.87 & 14.92 & $11.26 \pm 1.43$ & $8.99 \pm 0.62$ \\
\hline $\mathrm{NSp} * *$ & Number of spikelets per spike & $13.31 \pm 2.00$ & 8.33 & 18.33 & $13.42 \pm 2.00$ & $11.56 \pm 0.92$ \\
\hline TAL & Top awn length $(\mathrm{cm})$ & $3.20 \pm 0.58$ & 1.32 & 4.79 & $3.19 \pm 0.59$ & $3.40 \pm 0.35$ \\
\hline $\mathrm{SpL}^{b}$ & Spikelet length (mm) & $9.42 \pm 1.04$ & 7.12 & 12.23 & $9.42 \pm 1.06$ & $9.36 \pm 0.54$ \\
\hline $\mathrm{GL}^{b * * *}$ & Glume length (mm) & $6.67 \pm 0.50$ & 5.13 & 8.15 & $6.71 \pm 0.48$ & $6.04 \pm 0.46$ \\
\hline $\mathrm{GW}^{b * * *}$ & Glume width (mm) & $3.38 \pm 0.56$ & 2.28 & 5.33 & $3.29 \pm 0.43$ & $4.82 \pm 0.26$ \\
\hline $\mathrm{GT}^{b * * *}$ & Glume thickness (mm) & $0.24 \pm 0.03$ & 0.18 & 0.34 & $0.23 \pm 0.02$ & $0.31 \pm 0.02$ \\
\hline $\mathrm{GH}^{b * * *}$ & Glume height (mm) & $1.26 \pm 0.24$ & 0.88 & 1.96 & $1.23 \pm 0.21$ & $1.76 \pm 0.11$ \\
\hline AwL*** & Awn length (mm) & $5.50 \pm 6.17$ & 0 & 22.53 & $5.08 \pm 5.90$ & $12.27 \pm 6.58$ \\
\hline $\mathrm{LL}^{b * *}$ & Lemma length (mm) & $6.91 \pm 0.63$ & 5.62 & 8.72 & $6.94 \pm 0.63$ & $6.49 \pm 0.40$ \\
\hline $\mathrm{LW}^{b * * *}$ & Lemma width (mm) & $2.91 \pm 0.37$ & 2.10 & 4.02 & $2.87 \pm 0.34$ & $3.59 \pm 0.20$ \\
\hline $\operatorname{AnL} * * *$ & Anther length (mm) & $2.16 \pm 0.38$ & 1.49 & 3.48 & $2.14 \pm 0.38$ & $2.52 \pm 0.15$ \\
\hline $\mathrm{AnW}$ & Anther width (mm) & $0.61 \pm 0.07$ & 0.43 & 0.84 & $0.61 \pm 0.07$ & $0.64 \pm 0.04$ \\
\hline $\mathrm{AnT}^{* * *}$ & Anther thickness (mm) & $0.39 \pm 0.05$ & 0.23 & 0.51 & $0.38 \pm 0.05$ & $0.44 \pm 0.03$ \\
\hline PiL* & Pistil length (mm) & $1.37 \pm 0.17$ & 0.97 & 1.93 & $1.36 \pm 0.17$ & $1.47 \pm 0.08$ \\
\hline $\mathrm{PiW}^{* *}$ & Pistil width (mm) & $1.39 \pm 0.18$ & 0.98 & 1.83 & $1.38 \pm 0.18$ & $1.55 \pm 0.09$ \\
\hline $\mathrm{PiT}^{* * *}$ & Pistil thickness (mm) & $0.92 \pm 0.11$ & 0.67 & 1.48 & $0.91 \pm 0.11$ & $1.05 \pm 0.14$ \\
\hline $\mathrm{PaL}^{b *}$ & Palea length (mm) & $6.71 \pm 0.65$ & 5.48 & 8.68 & $6.72 \pm 0.66$ & $6.44 \pm 0.41$ \\
\hline $\mathrm{PaW}^{b * * *}$ & Palea width (mm) & $2.56 \pm 0.33$ & 1.87 & 3.58 & $2.52 \pm 0.29$ & $3.20 \pm 0.18$ \\
\hline AwL4 & Awn length of the 4th floret (mm) & $4.59 \pm 4.14$ & 0 & 19.28 & $4.56 \pm 4.20$ & $5.06 \pm 3.01$ \\
\hline ILSp & Internode length between spikelets (mm) & $8.70 \pm 0.87$ & 7.05 & 12.28 & $8.72 \pm 0.89$ & $8.47 \pm 0.57$ \\
\hline MCL*** & Main culm length $(\mathrm{cm})$ & $68.65 \pm 13.02$ & 42.01 & 111.98 & $67.71 \pm 12.69$ & $83.65 \pm 8.32$ \\
\hline $\mathrm{MCT}^{*}$ & Main culm thickness (mm) & $1.60 \pm 0.19$ & 1.10 & 2.10 & $1.57 \pm 0.19$ & $1.70 \pm 0.11$ \\
\hline IL1 & 1st internode length $(\mathrm{cm})$ & $25.65 \pm 5.96$ & 11.58 & 42.10 & $25.47 \pm 6.04$ & $28.59 \pm 3.28$ \\
\hline IL2 & 2nd internode length $(\mathrm{cm})$ & $14.42 \pm 3.66$ & 8.11 & 26.52 & $14.40 \pm 3.71$ & $14.76 \pm 2.70$ \\
\hline IL3 & 3rd internode length $(\mathrm{cm})$ & $10.46 \pm 2.04$ & 6.37 & 20.12 & $10.50 \pm 2.07$ & $9.90 \pm 1.36$ \\
\hline IL4* & 4th internode length $(\mathrm{cm})$ & $8.85 \pm 1.88$ & 5.20 & 16.07 & $8.77 \pm 1.86$ & $10.08 \pm 1.85$ \\
\hline
\end{tabular}

${ }^{a}$ Traits that show significant mean difference between the subspecies are indicated by $* P<0.05, * * P<0.01$, or *** $P<0.001$ (Student's $t$ test)

${ }^{b}$ Matsuoka et al. (2009)

$N=191$ in subspecies tauschil, $N=12$ in subspecies strangulata 
A

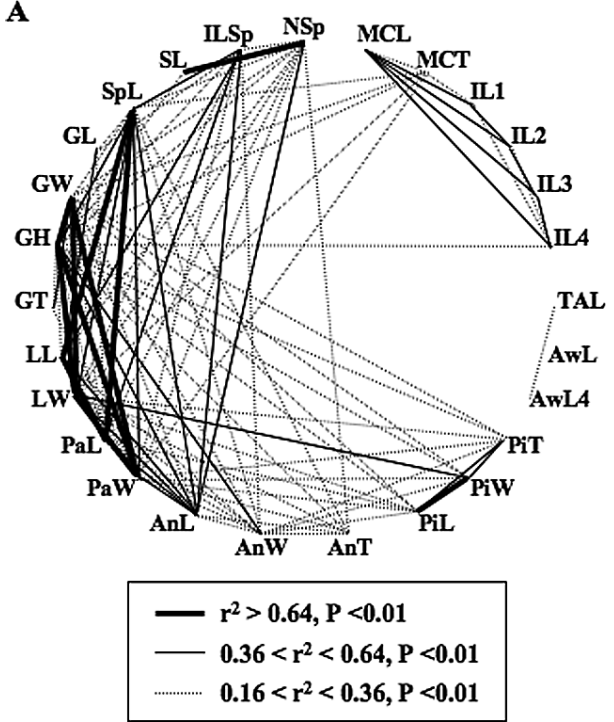

B

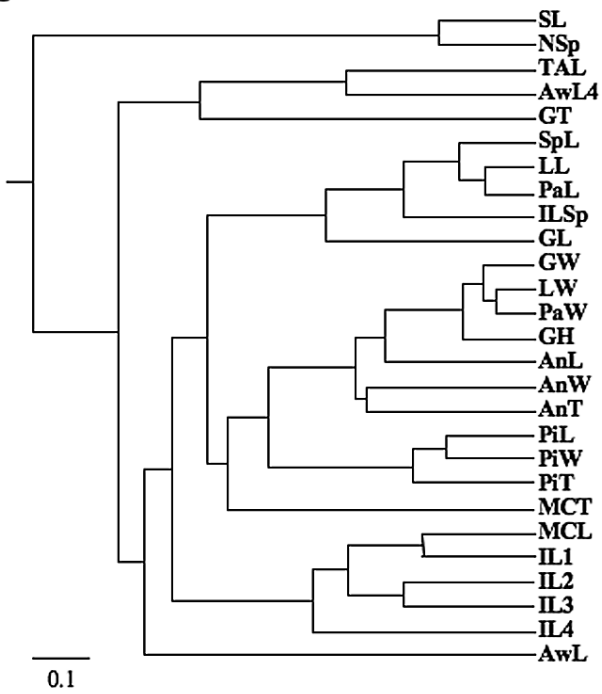

Fig. 2. Relationship of the 27 morphological traits. (A) The relation based on the Pearson coefficient values. Higher values are connected with thicker lines. (B) A dendrogram constructed by the UPGMA method based on the Pearson coefficients. The Pearson values (r) were transformed to the distance values $(\mathrm{d})$ as follows: $\mathrm{d}=(1-\mathrm{r})^{*} 100$.

variation was 50.2\% (36.6\% for $\mathrm{PC} 1$ and $13.6 \%$ for PC2). Next, PC analysis was done for spike and spikelet morphology-related traits. A total of 12 traits (SL, NSp, SpL, GL, GW, GT, GH, LL, LW, PaL, PaW and ILSp) were used as spike and spikelet morphology-related traits. The accumulated proportion of $\mathrm{PC} 1$ and $\mathrm{PC} 2$ in spike and spikelet morphological variation was $74.1 \%$ (53.6\% for $\mathrm{PC} 1$ and $20.5 \%$ for PC2). For the spike and spikelet morphologyrelated traits, the two subspecies formed separate clusters, and scatter plots showed subgroup formation (Fig. 3B). The subspecies strangulata cluster consisted of 12 accessions that were plotted with a positive value for $\mathrm{PC} 1$ and a value less than -2 for PC2 and did not overlap with the subspecies tauschii cluster. On the other hand, plant height-related traits such as SL, NSp, ILSp, MCL, MCT, IL1, IL2, IL3 and IL4 showed a widely distributed pattern in the scatter plots (Fig. 3C). The accumulated proportion of PC1 and PC2 in plant height-related trait variation was $66.9 \%$ (42.0\% for PC1 and 24.9\% for PC2). Subgroup formation was not found in PC analysis based on plant height-related traits.

Geographical clines of morphological traits based on multiple regression analysis

The morphological trait data set provided the opportunity to analyze the diversity patterns of Ae. tauschii morphology across its native range. We categorized the examined traits into four groups: plant height-related traits, spike-related traits, anther-related traits, and pistil-related traits. Plant height-related traits included MCL, IL1, IL2, IL3 and IL4. Spike-related traits included SL, NSp, ILSp and SpL. Anther- and pistil-related traits were based on their length, width and thickness. For evaluation of intraspecific variation, PC analysis was done for each trait group, and PC1 and PC2 values were calculated for each accession (Table 3).
Four accessions (CGN 10731, CGN 10732, CGN 10733 and CGN 10734) for which locality data were not available and six accessions (AT 47, AT 55, AT 60, AT 76, AT 80 and PI508264) that represented adventive populations were excluded from this analysis. As a result, PC1 and PC2 of plant height-related traits were found to capture $84.5 \%$ of the total variation $(69.92 \%$ for $\mathrm{PC} 1$ and $14.58 \%$ for PC2). PC1 and PC2 of spike-related traits captured $76.93 \%$ of the variation (52.64\% for PC1 and $24.29 \%$ for PC2). For anther- and pistil-related traits, $89.14 \%$ and $94.28 \%$ of the variation were respectively captured by $\mathrm{PC} 1$ (75.92\% for anther-related traits and $82.08 \%$ for pistil-related traits) and PC2 (13.22\% for anther-related traits and $12.20 \%$ for pistil-related traits). The PC1 and PC2 values were used for comparison between the geographical origins of the Ae. tauschii accessions and their morphological traits.

Previous studies reported that multiple regression analysis showed significant effects of latitude and longitude on PC1 values for spikelet-shape variation (Matsuoka et al. 2008, 2009). In this study, the diversity patterns of the four groups of morphological traits across the native range were analyzed by multiple regression analysis using the PC1 and PC2 values. In this analysis, the geographical coordinates of the sample collection sites (latitude and longitude) were used as the independent variables. On the basis of the eigenvectors, the $\mathrm{PC} 1$ and $\mathrm{PC} 2$ values of plant height-related traits and of anther- and pistil-related traits could be used as indices for size and form, respectively (Table 3 ). The PC1 value for spike-related traits could be used as an index for spike size and spikelet density, whereas the PC2 value could be used for spike size and spikelet size. PC1 values for plant height-related traits, spike-related traits, and anther-related traits were significantly influenced by longitude (Table 4 and Fig.4). Additionally, the PC2 value for anther-related 
A
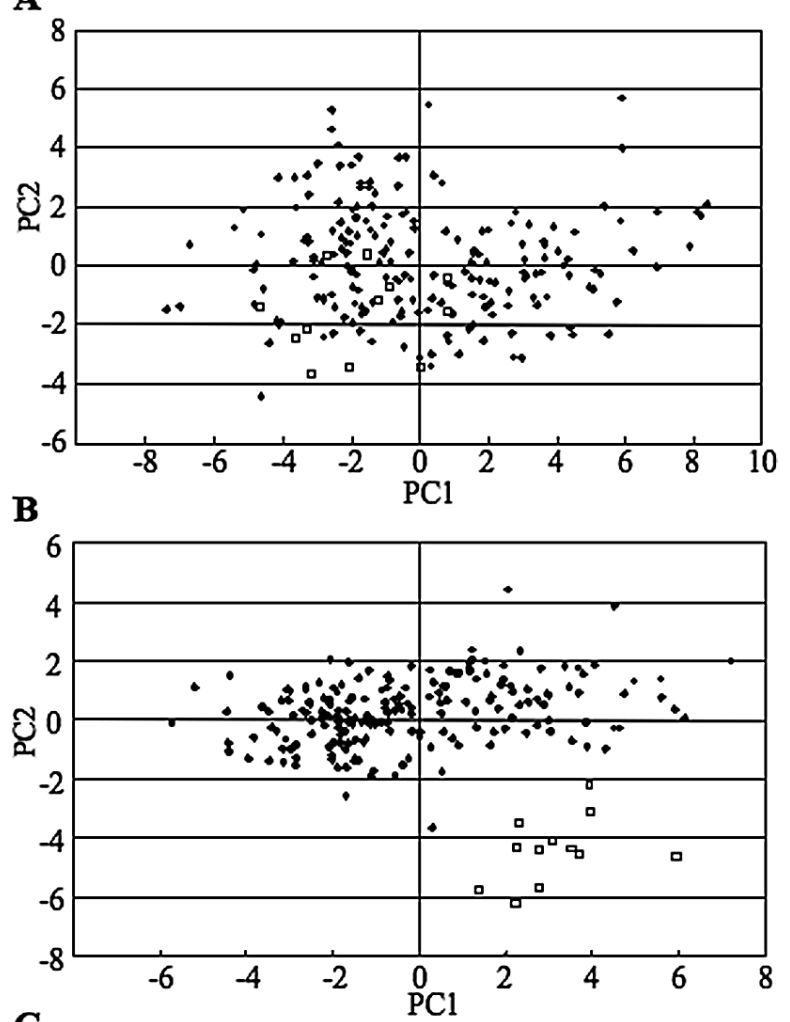

C

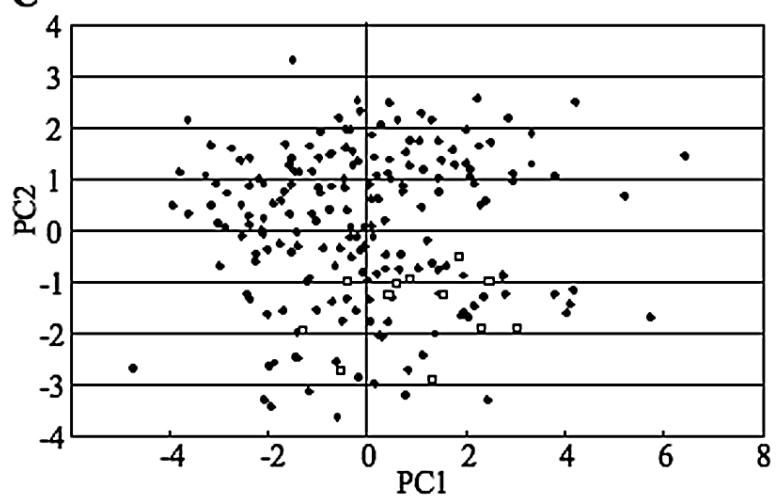

- ssp. tauschii

- ssp. strangulata

Fig. 3. Scatter plots based on the results of principal component (PC) analysis of Ae. tauschii morphological variation. White and black circles indicate subspecies strangulata and tauschii accessions, respectively. (A) PC values for all examined morphological traits. (B) PC values for twelve spike shape-related traits, SL, NSp, SpL, GL, GW, GT, GH, LL, LW, PaL, PaW and ILSp. (C) PC values for nine plant height-related traits, SL, NSp, ILSp, MCL, MCT, IL1, IL2, IL3 and IL4.

traits was significantly related to longitude. A significant effect of latitude on PC1 values of pistil-related traits was also observed.
Table 3. Eigenvectors for $\mathrm{PC} 1$ and $\mathrm{PC} 2$

\begin{tabular}{lrr}
\hline \hline Trait & PC1 & PC2 \\
\hline Plant height & & \\
MCL & 0.51 & -0.16 \\
IL1 & 0.41 & -0.70 \\
IL2 & 0.46 & -0.08 \\
IL3 & 0.42 & 0.58 \\
IL4 & 0.42 & 0.38 \\
Spike shape & & \\
SL & 0.56 & 0.47 \\
NSp & 0.67 & 0.15 \\
ILSp & -0.43 & 0.45 \\
SpL & -0.23 & 0.74 \\
Anther shape & & \\
AnL & 0.57 & -0.68 \\
AnW & 0.57 & 0.73 \\
AnT & 0.59 & -0.04 \\
Pistil shape & & -0.63 \\
PiL & 0.57 & -0.11 \\
PiW & 0.60 & 0.77 \\
PiT & 0.56 & \\
\hline
\end{tabular}

Relationship between morphological variations and chloroplast genome differentiation

The genealogical structure of the morphological variations was examined using $195 \mathrm{Ae}$. tauschii accessions. In our previous studies, four HG lineages, the HG7, HG9, HG16 and HG17 lineages, were defined based on chloroplast DNA differentiation (Matsuoka et al. 2008, 2009), and so these 195 accessions of Ae. tauschii were divided into the four HG lineages (Table 1 and Fig. 1). Accessions in the HG7 lineage were distributed across the entire range of natural habitats, and the eastward dispersal of Ae. tauschii was driven by the HG7 and HG16 lineages (Matsuoka et al. 2008). The HG9 lineage accessions were specific to western habitats, and the distribution area of five HG17 accessions was only found in Georgia.

Significant differences were found for some pairs of mean PC values (Table 5). The HG16 lineage exhibited a smaller mean than HG7 for PC2 values of plant height variation. For PC1 values of spike-shape variation, the accessions of the HG9 lineages had a significantly smaller mean than the accessions of the HG7 and HG16 lineages. Similarly, means of the HG16 lineage were significantly different from those of HG7 and HG9 lineages for the PC1 and PC2 values of anther-shape variation. Furthermore, there was a significant difference in the $\mathrm{PC} 1$ values of pistil-shape variation between the HG7 and HG9 lineages. No significant difference in the means was observed for the $\mathrm{PC} 1$ values of plant height variation and the $\mathrm{PC} 2$ values of spike-shape and pistil-shape variations.

\section{Discussion}

Natural morphological variation in Ae. tauschii

Iran is a center of genetic variation of Ae. tauschii 
Table 4. Multiple regression analysis of the effects of latitude and longitude on morphological traits using 195 accessions

\begin{tabular}{lllccrr}
\hline \hline Trait & Principal component & Source & Parameter estimate & Standard error & $t$ statistic & \multicolumn{1}{c}{$P$} \\
\hline Plant height & first & latitude & -0.015 & 0.06 & -0.30 & 0.7637 \\
& first & longitude** & -0.043 & 0.02 & -3.05 & 0.0026 \\
& second & latitude & 0.024 & 0.023 & 0.2979 \\
& second & longitude & -0.007 & 0.007 & -1.01 & 0.3146 \\
\hline Spike shape & first & latitude & -0.002 & 0.037 & -0.04 & 0.9662 \\
& first & longitude*** & 0.043 & 0.011 & 3.98 & $<0.0001$ \\
& second & latitude & 0.020 & 0.026 & 0.74 & 0.4597 \\
& second & longitude & -0.001 & 0.008 & -0.16 & 0.8719 \\
\hline Anther shape & first & latitude & -0.070 & 0.040 & -1.78 & 0.0772 \\
& first & longitude*** & -0.045 & 0.011 & -3.94 & 0.0001 \\
& second & latitude & 0.001 & 0.016 & 0.04 & 0.9686 \\
& second & longitude*** & 0.021 & 0.005 & 4.44 & $<0.0001$ \\
\hline Pistil shape & first & latitude*** & -0.160 & 0.041 & -3.91 & 0.11 \\
& first & longitude & 0.001 & 0.012 & 0.0001 \\
& second & latitude & 0.022 & 0.016 & 0.9119 \\
& second & longitude & -0.003 & 0.005 & 0.1791 \\
& & res & 0.5086 \\
\hline
\end{tabular}

This analysis was based on a bi-variate linear regression model using latitudinal and longitudinal locations (degrees) as independent variables. Significant effects are indicated by asterisks.

$\mathbf{A}$

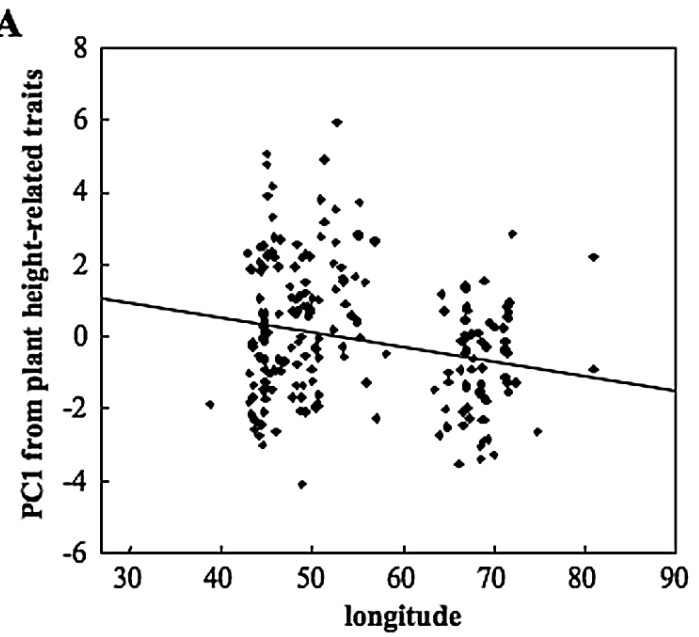

C

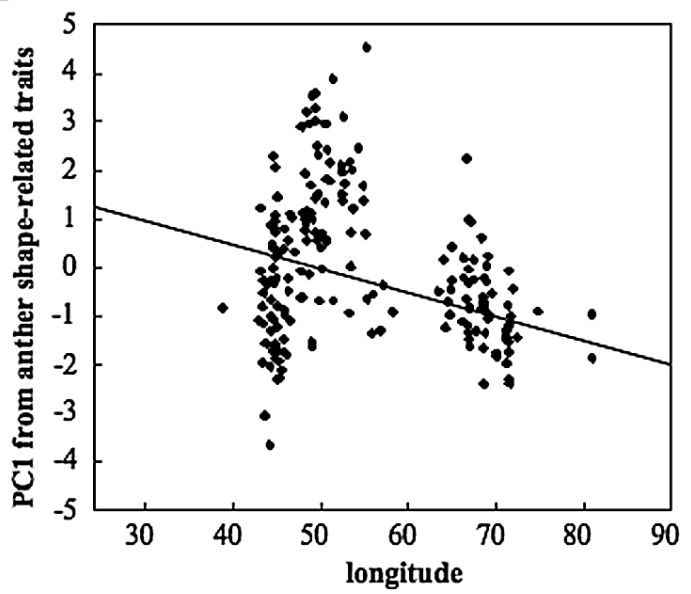

B

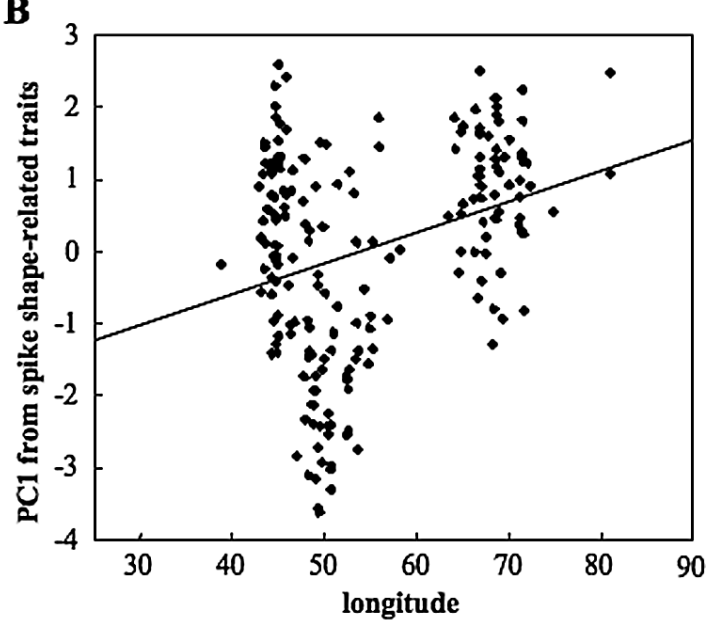

D

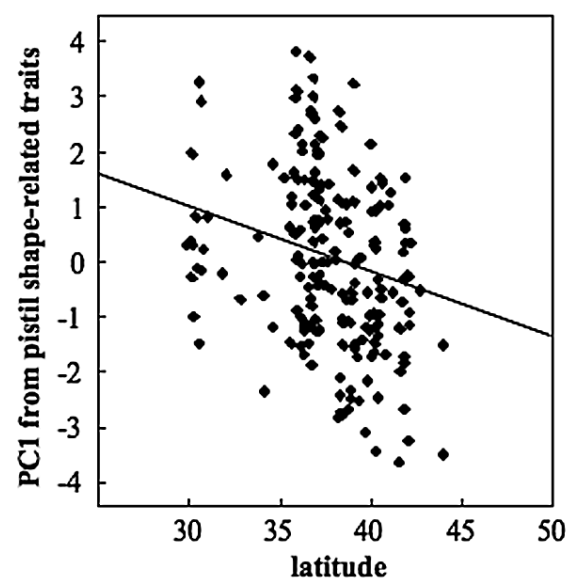

Fig. 4. Geographical clines of morphological trait variations $(N=195)$. (A) The relationship between longitude of origin and plant height. (B) The relationship between longitude and spike shape. (C) The relationship between longitude and anther shape. (D) The relationship between latitude of origin and pistil shape. 
Table 5. Comparison of mean principal component values of the four intraspecific lineages

\begin{tabular}{|c|c|c|c|c|c|}
\hline Trait & $\begin{array}{l}\text { Principal } \\
\text { component }\end{array}$ & Lineage & $N$ & Mean & $\begin{array}{c}\text { Standard } \\
\text { error }\end{array}$ \\
\hline \multirow{8}{*}{$\begin{array}{l}\text { Plant } \\
\text { height }\end{array}$} & \multirow[t]{4}{*}{ first } & HG7 & 114 & $0.25^{a}$ & 0.19 \\
\hline & & HG9 & 26 & $-0.01^{a}$ & 0.35 \\
\hline & & HG16 & 50 & $-0.49^{a}$ & 0.20 \\
\hline & & HG17 & 5 & $-0.72^{a}$ & 0.45 \\
\hline & \multirow[t]{4}{*}{ second } & HG7 & 114 & $0.14^{a b}$ & 0.08 \\
\hline & & HG9 & 26 & $-0.26^{b c}$ & 0.14 \\
\hline & & HG16 & 50 & $-0.26^{c}$ & 0.10 \\
\hline & & HG17 & 5 & $0.81^{a}$ & 0.39 \\
\hline \multirow{8}{*}{$\begin{array}{l}\text { Spike } \\
\text { shape }\end{array}$} & \multirow[t]{4}{*}{ first } & HG7 & 114 & $0.05^{b}$ & 0.13 \\
\hline & & HG9 & 26 & $-1.90^{c}$ & 0.20 \\
\hline & & HG16 & 50 & $0.85^{a}$ & 0.12 \\
\hline & & HG17 & 5 & $0.21^{a b}$ & 0.42 \\
\hline & \multirow[t]{4}{*}{ second } & HG7 & 114 & $-0.01^{a}$ & 0.09 \\
\hline & & HG9 & 26 & $0.10^{a}$ & 0.27 \\
\hline & & HG16 & 50 & $-0.02^{a}$ & 0.11 \\
\hline & & HG17 & 5 & $-0.02^{a}$ & 0.78 \\
\hline \multirow{8}{*}{$\begin{array}{l}\text { Anther } \\
\text { shape }\end{array}$} & \multirow[t]{4}{*}{ first } & HG7 & 114 & $-0.12^{b}$ & 0.15 \\
\hline & & HG9 & 26 & $1.54^{b}$ & 0.22 \\
\hline & & HG16 & 50 & $-0.62^{a}$ & 0.11 \\
\hline & & HG17 & 5 & $0.94^{a b}$ & 0.33 \\
\hline & \multirow[t]{4}{*}{ second } & HG7 & 114 & $-0.06^{b}$ & 0.06 \\
\hline & & HG9 & 26 & $-0.27^{b}$ & 0.12 \\
\hline & & HG16 & 50 & $0.32^{a}$ & 0.07 \\
\hline & & HG17 & 5 & $-0.37^{a b}$ & 0.10 \\
\hline \multirow{8}{*}{$\begin{array}{l}\text { Pistil } \\
\text { shape }\end{array}$} & \multirow[t]{4}{*}{ first } & HG7 & 114 & $-0.31^{b}$ & 0.16 \\
\hline & & HG9 & 26 & $0.76^{a}$ & 0.26 \\
\hline & & HG16 & 50 & $0.32^{a b}$ & 0.19 \\
\hline & & HG17 & 5 & $-0.14^{a b}$ & 0.49 \\
\hline & \multirow[t]{4}{*}{ second } & HG7 & 114 & $0.06^{a}$ & 0.06 \\
\hline & & HG9 & 26 & $-0.13^{a}$ & 0.10 \\
\hline & & HG16 & 50 & $-0.09^{a}$ & 0.08 \\
\hline & & HG17 & 5 & $0.35^{a}$ & 0.16 \\
\hline
\end{tabular}

For each principal component, mean values followed by the same letters were not significantly different $(P>0.05)$ (Tukey-Kramer's HSD test)

(Dudnikov and Goncharov 1993). In this study, Ae. tauschii exhibited large natural variations in the examined morphological traits. Scatter plots of PC analysis based on these morphological traits showed high levels of variation in each habitat from the western region to China. This finding indicated that the Ae. tauschii accessions in the non-Iranian habitats carried a large degree of genetic variation in morphological traits. The birthplace of common wheat has been assumed to be Transcaucasia or the south coastal region of the Caspian Sea (Tsunewaki 1966, Jaaska 1980, Nishikawa et al. 1980, Dvorak et al. 1998). Common wheat was derived from single or limited accessions of Ae. tauschii, and Ae. tauschii populations far from its birthplace were not involved in the formation of common wheat.

In this study, all examined traits except awn length- related traits showed continuous distribution. The awn length-related traits were correlated with each other, but independent of other morphological traits. These results suggested that most of the examined morphological traits may be determined by quantitative trait loci (QTL), whereas the awn length traits might be genetically controlled by a small number of major genes. There is little information about chromosomal location of the genetic loci generating the morphological diversity of Ae. tauschii. Detection of the QTLs and major genes that control these examined traits are required to understand the genetic framework for morphological diversification in Ae. tauschii.

Recognition of subspecies division in Ae.tauschii is controversial, because morphologically and genetically intermediate forms exist (Van Slageren 1994). In a previous study, we applied sensu stricto criteria for subspecies strangulata identification, defining it as having markedly moniliform spikes (Matsuoka et al. 2009). Our numerical analysis of spikelet morphology clearly distinguished the subspecies strangulata accessions from subspecies tauschii accessions. A similar result was obtained based on the natural genetic variation in the 12 traits SL, NSp, SpL, GL, GW, GT, GH, LL, LW, PaL, PaW and ILSp (Fig. 3B), although no clear subspecies division was observed in $\mathrm{PC}$ analyses using all the examined traits and plant height-related traits. Thus, the two Ae. tauschii subspecies, tauschii and strangulata, could be distinguished based on the PC1 and PC2 values of spikerelated morphological variation. The individual traits of spike and spikelet are in fact continuous in the Ae. tauschii populations (Van Slageren 1994, Dudnikov 2000), which resulted in questioning the biological significance of the subspecies division. Our results showed patterns of intraspecific morphological differentiation in the Ae. tauschii populations. As previously reported, GW is an important morphological trait to consider for the intraspecific differentiation of the two Ae. tauschii subspecies (Eig 1929, Dudnikov 2000, Dudnikov and Kawahara 2006). Our study also showed the importance of spikelet and floret shape-related traits including GW. GW was highly correlated with GH, LW and PaW (Fig. 2A), meaning that the same genes might control these traits. Further mapping of QTL controlling spikelet and floret morphology-related traits and subsequent gene isolation for the detected QTLs are required to address the molecular nature of differentiation in subspecies strangulata.

\section{Geographical clines of morphological variation}

The putative primary region of Ae. tauschii's origin is the Transcaucasus (Van Slageren 1994). From the Transcaucasus, the distribution area of Ae. tauschii expanded through eastward and southward dispersal. In this study, significant geographical clines were detected for each of the four morphological trait groups: plant height-related traits, spike-related traits, anther-related traits and pistil-related traits (Table 4 and Fig. 4). Our results indicated that (1) culm length tends to be short in the eastern region of the native range, (2) the spike tends to be long in the eastern 
region, (3) anthers tend to be small in the eastern region, and (4) pistils tend to be small in the northern region. Furthermore, in the eastern habitats, spikelet density per spike tended to be high, because eigenvectors of ILSp and SpL were negative for $\mathrm{PC} 1$ values of spike shape variation, in contrast to those of SL and NSp (Table 3).

Our previous study showed that the Ae. tauschii populations differentiated to the four chloroplast haplogroup lineages (Matsuoka et al. 2008). The largest HG7 lineage distributed across the current species' natural distribution range, whereas the HG9 and HG16 haplogroup lineages were confined to either the eastern or western habitats. The distribution area of five HG17 accessions was limited in Georgia. PC1 and PC2 values from the spike and anther shape-related traits in HG16 were significantly different from those in $\mathrm{HG} 9$, and significant difference of PC2 from the plant height-related traits was observed between HG16 and HG17 (Table 5). Therefore, the geographical clines for the morphological variations were consistent with results from the genealogical analysis using chloroplast DNA differentiation patterns.

The latitudinal cline found for the pistil size deserves a note. Matsuoka et al. (2009) analyzed spikelet-shape variation of 203 Ae. tauschii accessions and indicated that the spikelet size tends to be small in the southern region of the native range. Thus, the orientation of the pistil-size cline (small in the north, large in the south) appeared to be different from that of the spikelet-size cline (large in the north, small in the south). Reevaluation of the Matsuoka et al. (2009)'s work, however, identified an error that had been made in interpreting the data from multiple regression analysis. In that analysis, a negative correlation between the PC1 values for spikelet-shape variation and latitude was found. The correct interpretation of the data was that the spikelet size tends to be small in the northern region of the native range, because the $\mathrm{PC} 1$ values changed from low to high as spikelet size changed from small to large. Accordingly, Matsuoka et al. (2009)'s remark on the orientation of the latitudinal spikelet-size cline is wrong and needs to be corrected. When this correction is made, the orientation of the pistil-size and spikelet-size clines is the same: the accessions that are derived from northern regions of the native range tend to have small pistils and spikelets.

The longitudinal geographic clines found for several vegetative and reproductive organs suggest that, in the process of eastward dispersal from Transcaucasus, Ae. tauschii underwent morphological, genetic, and ecological diversification that produced the variation seen among today's natural populations. Previously, we reported the existence of significant longitudinal clines in variation of flowering time, which is a reproductive trait having a significant impact on fitness, implying that an early-flowering phenotype played an important role for Ae. tauschii to adapt to the eastern regions of its native range (Matsuoka et al. 2008). Similarly, small spikelets, short culms, long spikes with high spikelet density, and small anthers might reflect some type of adaptation to local conditions of the eastern habitats. The mechanisms underlying the morphological changes remain to be addressed, whereas relatively short vegetative and reproductive growth periods due to continental climate of the east might be a factor that affected the clinal patterns of morphological diversification. Determination of the genetic locus controlling such morphological changes may help understand the genetic mechanisms that underlie the adaptation to local conditions of the eastern habitats.

\section{Acknowledgements}

We thank J. Valkoun (ICARDA), J. Konopka (ICARDA), H. Bockelman (USDA), A. Graner (IPK), L. Visser (CGN), and K. Kato (Okayama Univ.) for Ae. tauschii accession seeds. This work was supported by grants from the Ministry of Education, Culture, Sports, Science and Technology of Japan (no. 17201045) and from the Ministry of Agriculture, Forestry and Fisheries of Japan (Genomics for Agricultural Innovation, TRC1003).

\section{Literature Cited}

Bandelt, H.J., P. Forster, B.C. Sykes and M.B. Richards (1995) Mitochondrial portraits of human populations using median networks. Genetics 141: 743-753.

Cadwell,K.S., J.Dvorak, E.S.Lagudah, E.Akhunov, M.C.Luo, P. Wolters and W.Powell (2004) Sequence polymorphism in polyploid wheat and their D-genome diploid ancestor. Genetics 167: 941-947.

Dudnikov, A.J. and N.P.Goncharov (1993) Allozyme variation in Aegilops squarrosa. Hereditas 119: 117-122.

Dudnikov,A.J. (1998) Allozyme variation in Transcaucasian population of Aegilops squarrosa. Heredity 80: 248-258.

Dudnikov, A.J. (2000) Multivariate analysis of genetic variation in Aegilops tauschii from the world germplasm collection. Genet. Resource Crop Evol. 47: 185-190.

Dudnikov, A.J. and T.Kawahara (2006) Aegilops tauschii: genetic variation in Iran. Genet. Resource Crop Evol. 53: 579-586.

Dvorak,J., M.C.Luo, Z.L.Yang and H.B.Zhang (1998) The structure of the Aegilops tauschii genepool and the evolution of hexaploid wheat. Theor. Appl. Genet. 97: 657-670.

Eig, A. (1929) Monographisch-kritische Übersicht der Gatteung Aegilops. Repertorium Specierum Novarum Rgni Vegetabilis. Beihefte 55: 1-228.

Garcia-Vallve, S., J.Palau and A.Romeu (1999) Horizontal gene transfer in glycosyl hydrolases inferred from codon usage in Escherichia coli and Bacillus subtilis. Mol. Biol. Evol. 9: 11251134.

Gianibelli, M.C., R.B. Gupta, D. Lafiandra, B. Margiotta and F. MacRitchie (2001) Polymorphism of high Mr glutenin subunits in Triticum tauschii: characterization by chromatography and electrophoretic methods. J. Cereal Sci. 33: 39-52.

Gianibelli, M.C., C.W.Wrigley and F. MacRitchie (2002) Polymorphism of low $\mathrm{Mr}$ glutenin subunits in Triticum tauschii. J. Cereal Sci. 35: 277-286.

Giles, R.J. and T.A.Brown (2006) GluDy allele variations in Aegilops tauschii and Triticum aestivum: implications for the origin of hexaploid wheats. Theor. Appl. Genet. 112: 1563-1572. 
Jaaska, V. (1980) Electrophoretic survey of seedling esterases in wheats in relation to their phylogeny. Theor. Appl. Genet. 56: 273284.

Kihara,H. (1944) Discovery of the DD-analyser, one of the ancestors of Triticum vulgare. Agric. Hortic. 19: 889-890.

Matsuoka,Y., N.Mori and T.Kawahara (2005) Genealogical use for chloroplast DNA variation for intraspecific studies of Aegilops tauschii Coss. Theor. Appl. Genet. 111: 265-271.

Matsuoka,Y., S.Takumi and T.Kawahara (2007) Natural variation for fertile triploid $F_{1}$ formation in allohexaploid wheat speciation. Theor. Appl. Genet. 115: 509-518.

Matsuoka,Y., S.Takumi and T.Kawahara (2008) Flowering time diversification and dispersal in central Eurasian wild wheat Aegilops tauschii Coss.: Genealogical and ecological framework. PLoS ONE3(9): e3138. Doi:10.1371/journal.pone.0003138

Matsuoka,Y., E.Nishioka, T.Kawahara and S.Takumi (2009) Genealogical analysis of subspecies divergence and spikelet-shape diversification in central Eurasian wild wheat Aegilops tauschii Coss. Plant Syst. Evol. 279: 233-244.

McFadden,E.S. and E.R.Sears (1944) The artificial synthesis of Triticum spelta. Rec. Genet. Soc. Am. 13: 26-27.

Nesbitt,M. and D.Samuel (1996) From staple crop to extinction? The archaeology and history of hulled wheat. In: Padulosi, S., K Hammer and J.Heller (eds.) Hulled Wheat: Promoting the Conservation and Use of Underutilized and Neglected Crops, Int. Plant Genet. Resources Institute, Rome, pp. 1-100.

Nishikawa,K., Y.Furuta and T.Wada (1980) Genetic studies on alphaamylase isozymes in wheat. III. Intraspecific variation in Aegilops squarrosa and birthplace of hexaploid wheat. Jpn. J. Genet. 55:
325-336.

Saeidi,H., M.R. Rahiminejad, S. Vallian and J.S. Heslop-Harrison (2006) Biodiversity of diploid D-genome Aegilops tauschii Coss. In Iran measured using microsatellites. Genet. Resource Crop Evol. 53: 1477-1484.

Thompson,J.D., D.G.Higgins and T.J.Gibson (1994) CLUSTAL W: improving the sensitivity of progressive multiple sequence alignment through sequence weighting, position-specific gap penalties and weight matrix choice. Nucl. Acids Res. 22: 4673-4680.

Tsunewaki,K. (1966) Comparative gene analysis of common wheat and its ancestral species. II. Waxiness, growth habit and awnedness. Jpn. J. Bot. 19: 175-229.

Van Slageren,M.W. (1994) Wild Wheats: A Monograph of Aegilops L. and Amblyopyrum (Jaub. \& Spach) Eig (Poaceae). Wageningen Agricultural University, Wageningen, pp. 326-344.

Yan, Y., S.L.K.Hsam, J.Yu, Y.Jiang and F.J.Zeller (2003a) Allelic variation of the HMW glutenin subunits in Aegilops tauschii accessions detected by Sodium Dodecyl Sulphate (SAS-PAGE), Acid Polyacrylamide Gel (A-PAGE) and Capillary Electrophoresis. Euphytica 130: 377-385.

Yan,Y., S.L.K.Hsam, J.Yu, Y.Jiang and F.J.Zeller (2003b) Genetic polymorphisms at Gli-Dt gliadin loci in Aegilops tauschii as revealed by acid polyacrylamide gel and capillary electrophoresis. Plant Breed. 122: 120-124.

Zhu,L.C., C.M.Smith, A.Fritz, E.Boyko, P.Voothuluru and B.S.Gill (2005) Inheritance and molecular mapping of new greenbug resistance genes in wheat germplasms derived from Aegilops tauschii. Theor. Appl. Genet. 111: 831-837. 